\title{
Chimeric Antigen Receptor (CAR) T Cell Therapy for Digestive Tumor
}

\author{
Chang $\mathrm{Wu}^{1, *}$, Jun $\mathrm{Wu}^{2}$ \\ ${ }^{1}$ Guangxi Medical University, No.22 Shuangyong Road, Qingxiu District, Nanning City, Guangxi Zhuang Autonomous Region,China \\ ${ }^{2}$ The First Affiliated Hospital of Chengdu Medical College, No.278 Baoguang Road, Xindu District, Chengdu City, Sichuan Province, \\ China
}

\begin{abstract}
Digestive tumors commonly include esophageal cancer, gastric cancer, liver cancer, pancreatic cancer. Most of which are malignant tumors. All of the tumors do strong harm to human body and seriously affect the physical and mental health of patients. With the change of modern dietary habits, the morbidity of digestive tumors is increasing year by year, and the threat to the society is increasingly intensified. Traditional treatments for digestive tumors include surgical resection, chemotherapy and radiotherapy, all of which can alleviate the symptoms to some extent, but there are still many drawbacks. Compared with traditional therapy, immunotherapy has better therapeutic effect and fewer adverse reactions. Immunotherapy is to activate the human immune system and kill tumor cells by its own immune function. In immunotherapy, CAR-T cell therapy, a kind of personalized therapy that takes effect through gene modification to obtain $\mathrm{T}$ cells carrying tumor antigen-specific receptor, occupies a leading position. At present, CAR-T cells have shown excellent results in the treatment of lymphoid and hematopoietic tumors, on the contrast, there are few studies on the treatment of solid tumors. In this article, we summarized the application of some CAR-T cells in solid tumors of the digestive system.
\end{abstract}

\section{Introduction}

CAR-T cell therapy is a personalized method through gene modification to obtain $\mathrm{T}$ cells that carry specific receptors for tumor antigens[1, 2]. Compared with conventional $\mathrm{T}$ cell antigens recognition, CAR's recognition of tumor antigens does not require $\mathrm{MHC}$ restriction, thus enabling $\mathrm{T}$ cells to achieve stronger antitumor destruction. CAR can be divided into three parts, antibody-like surface domain, transmembrane domain and intracellular signaling domain[3, 4]. According to the difference of intracellular signaling domain, there are actually 4 generations of CAR. First generation CAR consists of the $\mathrm{CD} 3 \zeta$ alone; the second generation includes additional costimulatory signaling domains (CD28 or $4-1 \mathrm{BB})$; the third generation combines two costimulatory domains together CD28 and 4-1BB); the fourth generation are additionally armored with genes that strengthen $\mathrm{T}$ cells' function (e.g. cytokines transgenes) (Figure 1)[3, 5, 6].

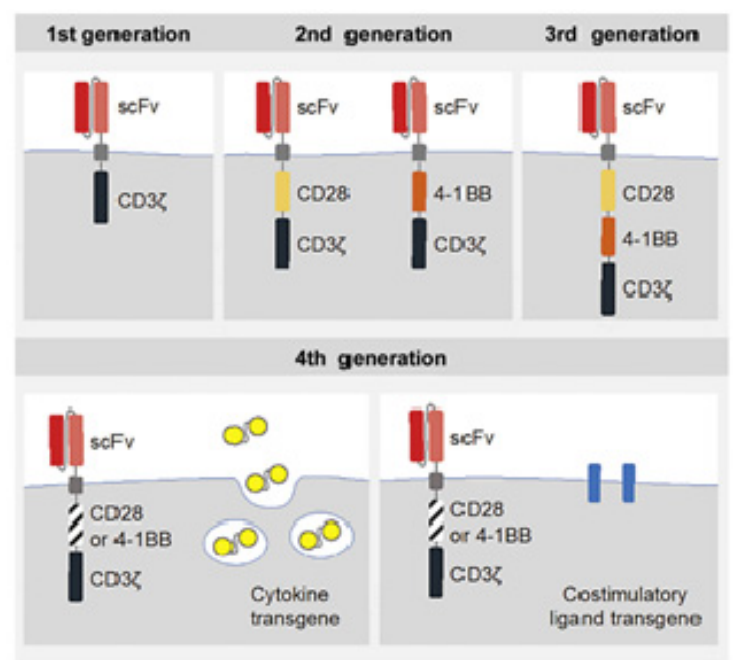

Figure 1. Generation of CAR-T[5]

\subsection{Target Receptors for Esophageal Cancer}

Esophageal cancer is a common tumor origin from esophageal epithelial cell with squamous cell carcinoma as the main type, known for its high morbidity and mortality[7]. The main clinical therapies for esophageal cancer nowadays are still the traditional surgery, radiation therapy and chemical therapy. It is true that the treatment methods could improve the patients' life quality, however, there are also defects like high

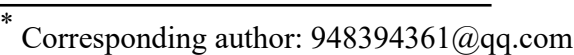


recurrence rate, low cure rate, adverse reactions, and many other shortcomings. Therefore, the demands for new treatments are gradually increasing. CAR-T cell therapy has attracted a lot of attention as a new anticancer method, and studies have found out that CAR-T cells have initial effectiveness in the treatment of esophageal cancer.

\subsection{Erythropoietin Producing Hepatocellular Receptor A2(EphA2)}

By immunofluorescence staining, immunohistochemical staining and WESTERN method, we can determine that the EphA2 only expresses on the cell membrane surface of esophageal cancer cells but not express specific receptors in normal tissue cell membranes. Through recognizing the specific receptor as the target, CAR-T cells can be designed to own specific anticancer effect on esophageal carcinoma cells. Flow cytometry analysis showed that about $57.6 \%$ of lentivirus vectors could be transferred into CAR $\mathrm{T}$ cells. For further testing of EphA2.CAR-T cell's effects, make normal T cells and EphA2.CAR T cell co-culture with CEA109 and TE-1 respectively, two types of esophageal cancer cell line, through calculating cancer mortality, crystal violet staining, lactate dehydrogenase(LDH) release quantity and cytokine release quantity, we can clearly conclude that: EphA2.CAR-T cells have stronger anticancer effect on the esophageal cancer cells than normal T cells[8].

\subsection{Human Epidermal Growth Factor Receptor 2(HER2)}

HER2 is a specific receptor highly expressed on the surface of TE-7 cells. The designed HER2-CAR T cells have been proved to have a specific anti-tumor effect on TE-7 cells, and can be transfected by Mir-143 to obtain stronger proliferation and secretion functions, so as to enhance its anti-tumor effect[9]. In addition, some studies have found that about $40 \%$ of esophageal cancer cells overexpress MSLN, while only little expression is found on the surface of normal mesothelial cells. The overexpressed specific molecule EpCAM was also found on the surface of esophageal squamous cell carcinoma cells. Currently, there are no studies having been conducted on MSLN and EpCAM in the treatment of esophageal cancer, but they may play an inestimable role as potential targets of CAR-T cell therapy for esophageal cancer in the future[10,11].

\section{Target Receptors for Gastric Cancer}

Gastric cancer is a malignant tumor originated from gastric mucosal epithelial cells. Its morbidity and mortality rank the fifth and third over the world, especially in Southeast Asia[12, 13]. At present, the common treatment for gastric cancer is a combination of surgical treatment with chemotherapy, radiotherapy and targeted therapy. However, the 5-year survival rate of patients with advanced gastric cancer under this treatment mode is less than $30 \%$. In addition, chemotherapy and radiotherapy may also cause damage to normal tissues and organs. As a result, there is a growing need for new treatment models with greater therapeutic power and fewer adverse effects. CAR-T cell therapy has been proven to have a definite therapeutic effect on gastric cancer[14].

\subsection{Claudin-18.2}

By immunohistochemical staining in the hSTMADE150CS4-01 and HSTM-ADE076MEt-01 microarrays, it was determined that Claudin-18.2 is a specific receptor on the surface of gastric carcinoma and metastatic gastric cancer cells. However, after adding anti-claudin-18.2 antibody into normal gastric tissue sections, immunostaining revealed claudin-18.2 also exists on the surface of normal gastric cells. Therefore, CAR-T cells targeting this receptor may own a potential risk of "on target off tumor". Three specific CAR-T cells, HU8E5-28Z, Hu8E5-BBZ and Hu8E5-21-28Z were designed and co-cultured together with 293T-Claudin18.2 and gastric cancer cell lines AGS and BGA-823 respectively. By counting the lysis rate of cancer cells, tumor volume and cytokines released of IL-2, a conclusion could be drawn: The three types of claudin18.2-CAR-T cells all have a strong antitumor effect on gastric cancer cells, among which, the cytokine secretion capacity of HU8E5-21-28Z was significantly stronger than that of Hu8E5-28Z. In addition, through peripheral blood CD4+/CD8+T cell count, it was clear that HU8E521-28Z had a strong proliferation ability. In order to exclude the possibility of "on target off tumor", HU8E521-28z was injected into PDX model mice with gastric cancer, the weight change curve and immunostaining were recorded, and then compared with the normal control group. It was obvious that Hu8E5-21-28Z has no cytotoxicity on normal gastric tissue[15].

\subsection{HER2}

As mentioned above, HER2 plays a role as a receptor specifically expressed on the surface of esophageal cancer cells, at the same time, it has been confirmed that specific expression of HER2 also exists on the surface of gastric cancer cells. chA21-4-1BBZ-CAR-T cells are CAR-T cells with specific antitumor effect on HER2positive tumor cells. chA21-4-1BBZ-CAR-T cells were co-cultured with SKOV3, NCI-N87, HGC-27 and other cell lines, and the total number of $\mathrm{T}$ cells and the secretion amount of cytokines were measured. The analysis results showed that the proliferation ability of chA21-4-1BBZ-CAR-T cells was almost the same as that of normal T cells, but some cytokines (TNF-A, IL-2, IFN-y) are secreted abundantly. In NOD/SCID mice model two bilateral symmetry parts were subcutaneous implanted with MKN - 28 and NCI - N87 respectively, 40th and 45th day after tumor implantation, injected chA21-4-1BBZ-CAR-T cells into the mice, and then recorded the tumor volume, peripheral blood $\mathrm{T}$ cell count and immunohistochemical staining. Conclusion can be drawn: chA21-4-1BBZ-CAR-T cells have 
obvious antitumor effect to NCI - N87 and nearly no effect to MKN-28. In addition, subcutaneous implantation of NCI-N87 in NOD/SCID model mice was followed by injection of chA21-4-1BBZ-CAR-T cells on the 7th and 10th days after tumor implantation, then measured by abdominal laparotomy and survival statistics, the results showed that chA21-4-1BBZ-CAR-T cells could significantly improve the survival rate and survival days of gastric cancer models and inhibit peritoneal metastasis of gastric cancer cells[16, 17].

\subsection{Folate receptor 1 (FOLR1)}

FOLR1 has been proven to be overexpressed on the surface of more than $1 / 3$ gastric cancer cells, while not expressed on the surface of normal gastric tissue cells, making FOLR1 effective as a target for immunotherapy for gastric cancer. FOLR1-CAR and FOLR1 were expressed on the surfaces of Jurkat cells and K562 cells respectively by lentivirus vector transfection, and the two types of cells were co-cultured. Measurement of IL2 secretion quantity confirmed that FOLR1-CAR could enable the cells to obtain stronger cytokine secretion capacity. FOLR1-CAR Jurkat cells were co-cultured with FOLR1-positive gastric cancer cell lines and FOLR1-negative gastric cancer cell lines, and IL-2 secretion was measured. It was concluded that FOLR1CAR had a highly specific and lethal effect on FOLR1. After the function of FOLR1-CAR was confirmed, the designed FOLR1-CAR-T cells were co-cultured with the gastric cancer cell lines, and the $\mathrm{T}$ cell count and cytokine secretion quantity were measured. It can be concluded that FOLR1-CAR-T cells have highly specific and antitumor effect on FOLR1, but the proliferation ability of CAR-T cells was not changed. In addition, ZAAD-CMK, anti-FasL and anti-TNFa were added to the culture environment of FOLR1-CAR-T cells and gastric cancer cell lines, and the cell lysis rate was calculated after a certain period of culture. It was obviously that the cytotoxic effect of FOLR1-CAR-T cells was mainly achieved by granzyme B. WESTERN Blot was used to measure the content of intracellular correlation protein and corresponding phosphorylated products. Quantitative analysis showed that the target cells of FOLR1-CAR-T cell lysis were transduced through the ZAP70 pathway. WESTERN Blot was also used to measure the contents of related proteins in the lysed cells, and contrastive analysis showed that FOLR1-CAR-T cells play an anti-tumor role by inducing apoptosis of target cells[18].

\subsection{Mesothelin (MSLN)}

Immunohistochemical staining showed that MSLN was expressed on the surface of gastric cancer cell membrane but not on the surface of normal tissue cells. M28Z10-T cells were designed with this specific receptor to have specific antitumor effect on gastric cancer cells. Experimental analysis showed that the proliferation of M28Z10-T cells in vitro was no different from that of normal $\mathrm{T}$ cells, but compared with normal $\mathrm{T}$ cells,
M28Z10-T cells had a stronger specific anti-tumor effect on gastric cancer cells and could induce a stronger immune response. In addition, tumor size, weight and peripheral blood $\mathrm{T}$ cell ratio of different M28Z10-T cell injection methods were examined respectively in the experiment, and it can be clearly concluded that the antitumor effect of peritumor(p.t) injection is significantly stronger than that of intravenous(i.v) injection, which will have a certain guiding significance for future clinical application[19, 20].

\section{Target Receptors for Liver Cancer}

Liver cancer includes primary cancer and metastatic cancer, most of which are primary liver cancer. Primary liver cancer originates from hepatocytes and intrahepatic bile duct epithelial cells. In China, liver cancer represents the second most diagnosed cancer and the fourth most cancer-associated death. At present, the common treatment of liver cancer is still mainly surgical treatment, such as surgical resection, radiofrequency ablation, embolization procedures and so on. However, the postoperative cure rate is low with high recurrence rate, so the therapeutic effect is not ideal. Chemotherapy and radiotherapy can also play a certain role in the treatment of liver cancer, but the effect is not good as well. CAR-T cell therapy, characterized by its high specificity and high efficiency, is expected to become a major role in the future treatment of liver cancer[21, 22].

\subsection{Alpha-fetoprotein (AFP)}

AFP is an intracellular receptor specifically expressed in hepatocellular carcinoma cells and therefore cannot be directly recognized by conventional CAR. Through MHC-I processing on the AFP into the form of AFP/MHC complex expressed on liver cancer cell surface, AFP can be specifically designed for the target of AFP-CAR-T cells. AFP-CAR-T cells have specific anti-tumor effect on liver cancer cells without specific effect on non-liver cancer cells, and have strong cytokine secretion ability. In vivo experiments on hepatocellular carcinoma model showed that intratumor(i.t) injection had more obvious anti-tumor effect than i.t injection, and the anti-tumor effect was enhanced with the increase of CAR-T cell dose[23].

\subsection{Carcinoembryonic Antigen (CEA)}

CEA is a kind of receptor expressed on the surface of liver cancer cells. The experiment attempted to achieve the purpose of treating liver cancer by producing CEACAR-T cells that can specifically bind to CEA, but the therapeutic effect was not ideal. Further studies have found that there are liver $\mathrm{CD} 11 \mathrm{~b}+\mathrm{Gr}-1+$ myeloid-derived suppressor cells(L-MDSC) in liver cancer tissues, which may lead to poor therapeutic effect by inhibiting the activity of CAR-T cells. In the experiment, anti-LMDSC antibody was added in the co-culture environment of tumor cells and CAR-T cells, and it turned out that the anti-tumor effect of CAR T cells was 
significantly improved. Further studies on the mechanism of L-MDSC revealed that the inhibition was related to the presence of GM-SCF and STAT3. Both of the above factors promoted the inhibition of CAR-T cells by up-regulating the expression level of PD-L1 on the surface of L-MDSC cells. In vivo experiment, corresponding antibodies were added and co-cultured together, and the results showed that each antibody could improve the anti-tumor effect of CAR-T cells[24, 25].

Additionally, glypican-3, MUTc-1, EpCAM have been confirmed to be potential target receptors for the treatment of liver cancer[21].

\section{Target Receptors for Pancreatic Cancer}

Pancreatic cancer is a malignant tumor derived from pancreatic ductal epithelium and follicular cells. It is characterized by high degree of malignancy, insidious onset and rapid progression, with poor prognosis and frequent recurrence and metastasis. In recent years, its morbidity and mortality have been rising globally. At present, the commonly used treatment for pancreatic cancer is still the traditional treatment while its five-year survival rate is only $5 \%$, and the five-year survival rate of patients undergoing radical resection is no more than $20 \%$. CAR-T cell therapy has the advantages that the above therapies do not have, which provides a new solution for the treatment of pancreatic cancer[26-28].

\subsection{CD47}

CD47 is an overexpressed receptor on the surface of various tumor cells, but it is also expressed in small amounts in some normal tissues. Murine CD47-CAR-T cells with specific affinity were designed to target CD47 in the experiment, and relevant in vitro and in vivo experiments were carried out. The experimental results showed that the CAR-T cells had obvious specific cytotoxicity to CD47. Subsequently, human CD47-CAR$\mathrm{T}$ cells were generated in the laboratory and related experiments were carried out then, leading to the same experimental results as murine CAR-T cells. In addition, human CD47-CAR-T cells were respectively co-cultured with lymphoma cells and normal tonsil cells. Immunohistochemical staining showed that there was no 'on target/off tumor' phenomenon in the CAR-T cells[29].

Other studies have shown that CD24, HER2 and prostate-stem cell antigen are all specific receptors on the surface of pancreatic cancer cells, which have certain reference value for the treatment of pancreatic cancer[26].

\section{Conclusion}

With the changes in people's dietary habits, the morbidity of digestive tumors has increased year by year, making patients suffer from the disease. At present, the conventional treatment of digestive system tumors is still the traditional treatment, including surgical resection, radiotherapy, chemotherapy and immunotherapy. Traditional treatment can relieve the symptoms of patients to a certain extent, but at the same time, it also has obvious disadvantages such as high recurrence rate, damage to normal tissues and long period of treatment. As a result, there is a growing need for new treatments. Among them, CAR-T cell therapy, as a new treatment method, has been preliminarily prepared as a potential clinical treatment, attracting much attention in the academic field. The therapeutic effect of CAR-T cells in leukemia, lymphoma and other tumors has been fully demonstrated, but there are few studies on the treatment of solid tumors by CAR-T cells, related clinical trials are extremely scarce. In this article, we summarized the relevant data of CAR-T cell therapy in different digestive tumors and introduced some target receptors and the therapeutic effects.

The manufacturing process of CAR-T cells usually consists of the following steps: 1) Identify and recognize receptors specifically expressed on the surface of tumor cells; 2) Find antibodies that can bind to the target receptor; 3) The corresponding CAR is made based on the region where the antibody binds to the receptor; 4) Insert CAR chimerism into the virus vector; 5) $\mathrm{T}$ cells are selected from the patients by flow cytometry, then, the viral vector is implanted into the T cells, and finally, CAR-T cells are selected; 6) Detect whether the CAR exists on the surface of CAR-T cells through various means like mRNA, immunohistochemistry or WESTERN; 7) Relevant in vivo and in vitro experiments are conducted to verify whether CAR-T cells have specific anti-tumor effects (Figure 2)[5].

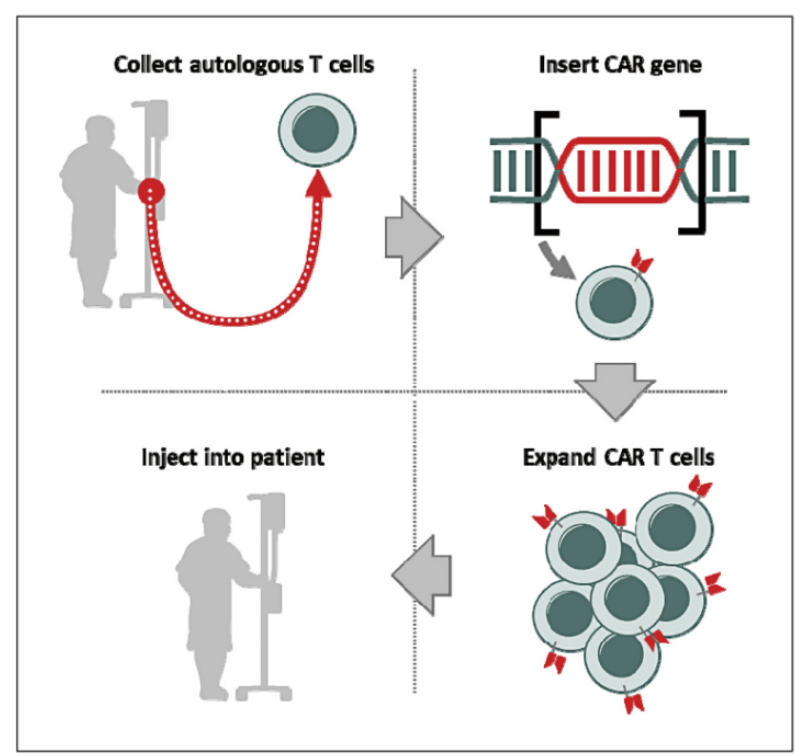

Figure 2. Scheme of CAR-T therapy[5]

However, CAR-T cell therapy has also revealed some drawbacks in related experiments. 1) Cytokine release syndrome (CRS) is the most common adverse reaction in CAR-T cell therapy. It is due to the proliferation of CAR-T cells in the body after contact with specific receptors on the surface of tumor cells, and a large number of cells are produced Factors, causing a 
series of damage to the patient's normal tissues. The clinical manifestations of CRS are more diverse, usually manifested as high fever, fatigue, myalgia, nausea, tachycardia, progressive hypotension, etc. The severe cases can endanger the life of the patient. CRS often appears within 1 week after cell infusion, and reaches its peak within 1 to 2 weeks after cell infusion. When the patient is found to have related symptoms, treatment should be taken immediately. Mild patients can be closely monitored, symptomatic treatment, supportive treatment, etc., while for middle-to-high patients, IL-6 inhibitors (tocilizumab) or glucocorticoids should be used. If the symptoms still do not improve, cyclophosphamide can be used Or anti-T cell therapy such as alemtuzumab; 2) "on target/off tumor" is a potential phenomenon in CAR-T cell therapy, which means that the receptor as a CAR-T cell target is not only expressed on the surface of tumor cells, It is also expressed on the surface of some normal cells, so that CAR-T cells can cause irreversible damage to normal tissues while having anti-tumor effects. In order to avoid such situations, tumor cell surface receptors with higher specificity should be sought as targets to eliminate the possibility of CAR-T cells causing damage to normal tissues; 3) The tumor microenvironment, that is, the inner life of tumor cells In addition to tumor cells, the environment also includes various cells such as fibroblasts and inflammatory cells around it, as well as intercellular substance, micro vessels, and biomolecules infiltrating the tumor. In the tumor microenvironment, lymphocytes and other immune cells can mediate immune suppression, enabling tumor cells to achieve immune escape and promote their malignant progress. In addition, pro-angiogenic factors can also promote the growth and metastasis of tumors by accelerating the formation of micro vessels in tumors. Therefore, in order to obtain the maximum therapeutic effect, the tumor microenvironment should be clinically classified to guide clinical treatment, such as using peritumoral (pt) or intratumoral (it) injection to replace traditional intravenous (iv) injection; 4 ) At present, the cost of manufacturing CAR-T cells is relatively high, and the process is also cumbersome and time-consuming. Considering the income level of ordinary families, not all patients are financially capable of receiving the treatment. When the technology matures, the manufacturing process will gradually be simplified, and the related costs will gradually decrease. By then, more patients will be able to provide better treatment.

\section{Reference}

1. Curran, K.J., H.J. Pegram, and R.J. Brentjens, Chimeric antigen receptors for $\mathrm{T}$ cell immunotherapy: current understanding and future directions. J Gene Med, 2012. 14(6): p. 405-15.

2. Haji-Fatahaliha, M., et al., CAR-modified T-cell therapy for cancer: an updated review. Artif Cells Nanomed Biotechnol, 2016. 44(6): p. 1339-49.

3. Pettitt, D., et al., CAR-T Cells: A Systematic Review and Mixed Methods Analysis of the Clinical
Trial Landscape. Molecular Therapy, 2018. 26(2): p. 342-353.

4. Khalil, D.N., et al., The future of cancer treatment: immunomodulation, CARs and combination immunotherapy. Nature Reviews Clinical Oncology, 2016. 13(5): p. 273-290.

5. Subklewe, M., M. von Bergwelt-Baildon, and A. Humpe, Chimeric Antigen Receptor T Cells: A Race to Revolutionize Cancer Therapy. Transfus Med Hemother, 2019. 46(1): p. 15-24.

6. van der Stegen, S.J.C., M. Hamieh, and M. Sadelain, The pharmacology of second-generation chimeric antigen receptors. Nature Reviews Drug Discovery, 2015. 14(7): p. 499-509.

7. Accordino, G., et al., From Interconnection between Genes and Microenvironment to Novel Immunotherapeutic Approaches in Upper GastroIntestinal Cancers-A Multidisciplinary Perspective. Cancers (Basel), 2020. 12(8).

8. Shi, H., et al., EphA2 chimeric antigen receptormodified $\mathrm{T}$ cells for the immunotherapy of esophageal squamous cell carcinoma. J Thorac Dis, 2018. 10(5): p. 2779-2788.

9. Zhang, T., et al., miR-143 Regulates Memory T Cell Differentiation by Reprogramming $\mathrm{T}$ Cell Metabolism. J Immunol, 2018. 201(7): p. 2165-2175.

10. McGowan, E., et al., PD-1 disrupted CAR-T cells in the treatment of solid tumors: Promises and challenges. Biomed Pharmacother, 2020. 121: p. 109625.

11. Kiesgen, S., et al., Chimeric Antigen Receptor (CAR) T-Cell Therapy for Thoracic Malignancies. J Thorac Oncol, 2018. 13(1): p. 16-26.

12. Zhao, W., et al., The killing effect of novel bispecific Trop2/PD-L1 CAR-T cell targeted gastric cancer. Am J Cancer Res, 2019. 9(8): p. 1846-1856.

13. Zhang, Z., et al., Modified CAR T cells targeting membrane-proximal epitope of mesothelin enhances the antitumor function against large solid tumor. Cell Death Dis, 2019. 10(7): p. 476.

14. Yang, L., Y. Wang, and H. Wang, Use of immunotherapy in the treatment of gastric cancer. Oncol Lett, 2019. 18(6): p. 5681-5690.

15. Jiang, H., et al., Claudin18.2-Specific Chimeric Antigen Receptor Engineered $\mathrm{T}$ Cells for the Treatment of Gastric Cancer. J Natl Cancer Inst, 2019. 111(4): p. 409-418.

16. Han, Y., et al., Antitumor effects and persistence of a novel HER2 CAR $T$ cells directed to gastric cancer in preclinical models. Am J Cancer Res, 2018. 8(1): p. 106-119.

17. Song, Y., et al., Effective and persistent antitumor activity of HER2-directed CAR-T cells against gastric cancer cells in vitro and xenotransplanted tumors in vivo. Protein Cell, 2018. 9(10): p. 867-878.

18. Kim, M., et al., Folate receptor 1 (FOLR1) targeted chimeric antigen receptor (CAR) $\mathrm{T}$ cells for the 
treatment of gastric cancer. PLoS One, 2018. 13(6): p. e0198347.

19. Bębnowska, D., et al., CAR-T Cell Therapy-An Overview of Targets in Gastric Cancer. J Clin Med, 2020. 9(6).

20. Sotoudeh, M., et al., MSLN (Mesothelin), ANTXR1 (TEM8), and MUC3A are the potent antigenic targets for CAR $\mathrm{T}$ cell therapy of gastric adenocarcinoma. J Cell Biochem, 2019. 120(4): p. 5010-5017.

21. Chen, Y., et al., Chimeric antigen receptorengineered T-cell therapy for liver cancer. Hepatobiliary Pancreat Dis Int, 2018. 17(4): p. 301309.

22. Xia, A.L., et al., Chimeric-antigen receptor T (CART) cell therapy for solid tumors: challenges and opportunities. Oncotarget, 2017. 8(52): p. 9052190531.

23. Liu, H., et al., Targeting Alpha-Fetoprotein (AFP)MHC Complex with CAR T-Cell Therapy for Liver Cancer. Clin Cancer Res, 2017. 23(2): p. 478-488.

24. Zhang, C., et al., Phase I Escalating-Dose Trial of CAR-T Therapy Targeting CEA $(+)$ Metastatic Colorectal Cancers. Mol Ther, 2017. 25(5): p. 12481258.

25. Burga, R.A., et al., Liver myeloid-derived suppressor cells expand in response to liver metastases in mice and inhibit the anti-tumor efficacy of anti-CEA CAR-T. Cancer Immunol Immunother, 2015. 64(7): p. 817-29.

26. DeSelm, C.J., et al., CAR T-cell therapy for pancreatic cancer. J Surg Oncol, 2017. 116(1): p. 63-74.

27. Akce, M., et al., The Potential of CAR T Cell Therapy in Pancreatic Cancer. Front Immunol, 2018. 9: p. 2166.

28. Watanabe, K., et al., Pancreatic cancer therapy with combined mesothelin-redirected chimeric antigen receptor $\mathrm{T}$ cells and cytokine-armed oncolytic adenoviruses. JCI Insight, 2018. 3(7).

29. Golubovskaya, V., et al., CD47-CAR-T Cells Effectively Kill Target Cancer Cells and Block Pancreatic Tumor Growth. Cancers (Basel), 2017. $9(10)$. 